\title{
The capture of comets by swing-by
}

\author{
ANTONIO FERNANDO BERTACHINI DE ALMEIDA PRADO ${ }^{1}$ \\ and ROGER A. BROUCKE ${ }^{2}$ \\ ${ }^{1}$ Instituto Nacional de Pesquisas Espaciais, 12227-010 São José dos Campos, SP, Brazil \\ 2 Department of Aerospace Engineering, University of Texas at Austin \\ E-mails: prado@dem.inpe.br/broucke@uts.cc.utexas.edu
}

\begin{abstract}
In this paper, numerical simulations are made, using the restricted three-body problem as the mathematical model, to calculate the effects of a swing-by with the planet Jupiter in the orbit of a comet. In particular, the orbital elements of the comets are calculated after the passage by Jupiter, the distribution obtained is shown and the probability of capture is calculated as a function of the Jacobian constant of the comet. The results show that the probability of capture goes from $0.2 \%$ to $3.6 \%$, depending on the value of the Jacobian constant of the comet. The details of this mechanism and some trajectories are shown. It is also concluded that more than $95 \%$ of the captured comets have pericenter distance less than $5 \mathrm{AU}$ (what makes them visible) and that about $70 \%$ of them are short-period comets.
\end{abstract}

Mathematical subject classification: $70 \mathrm{~F} 07,70 \mathrm{~F} 16,70 \mathrm{~F} 15$.

Key words: comets, swing-by, restricted problem.

\section{Introduction}

The comets are among the smallest bodies known in the Solar System, but they are also one of the most important and interesting topics of research in today's celestial mechanics. Comets are believed to carry material from the time that the Solar System was formed, which means that a detailed study of that material could help us to answer many basic questions about this important process. There are also some speculations about connections between the impact of the comets and the origin of life on Earth. The comets are so small, that they are 
only detected when they are passing near their pericenter. This fact makes the estimation of their quantity a very difficult and unsolved problem. The literature almost agrees that a good estimate for a lower limit for their population is about $10^{11}$ comets related to the Solar System, but several researchers believe that they are much more numerous. The actual observations show that three or four new comets are observed in the interior Solar System every year.

The origin of the comets is still not explained. They can be formed in the interstellar space and then they are captured by the Solar System [1,2] or they can be formed in the Solar System and then they are expelled to the interstellar space. One of the most popular ideas about the origin of the comets is the existence of a large cloud of comets around the Solar System, called the "Oort Cloud", with a total of $10^{11}$ comets at distances up to $10^{5} \mathrm{AU}[3,4]$.

The present research, that is based in Prado [5], has the goal of giving a contribution to the problem of capture and escape of comets caused by a close encounter with the planet Jupiter. The topics of escape and capture of comets have been discussed in the literature for a long time. In the majority of the papers, a close approach with a large planet, usually Jupiter, is the core of the mechanism of capture and escape. The dynamics are explained in papers as old as Russell [6] and Woerkom [7], who derived an expression to calculate the effects of the close approach between Jupiter and one comet. More details about the study of comets can be found in papers by Whipple [8], Everhart [9,10], Joss [11], Öpik [12], Marsden, Sekanina and Yeomans [13], Yabushita [14], Hills [15], Fernández [16], Bailey [17,18,19], Duncan and Quinn [20], Bailey and Stagg [21,22], Petroski and Broucke [23], Stagg and Bailey [24], Chirov and Vecheslavov [25], Yabushita and Tsujii [26], Wetherill [27], Emel'yanenko [28], Levison and Duncan [29], Fernández and Gallardo [30], Emel'yanenko and Bailey [31,32].

In the present paper a new set of initial conditions for the numerical integration and a new classification of the orbits before and after the close approach are introduced. With these two new concepts, it is possible to perform a study of the evolutions of the orbits and to calculate the distribution of the comets after the close approach. This can give us some conclusions about the model used. 


\section{Mathematical Model and Algorithm}

The present section gives a simple example that illustrates the basic principle of energy increase or decrease during a flyby of a moving body. It justifies the following fundamental conclusions: when a particle has a close approach with a receding massive body, it gains energy, while, if the massive body is approaching, the particle loses energy.

It is assumed that $Q$ is a massive planet and $P$ is the comet with negligible mass. In other words $P$ has no effect on the motion of $Q$. The comet $P$ has a close approach with the planet $Q$. A correct treatment of the problem requires the restricted three-body problem, that is described later. The present treatment is somewhat simplified, but it illustrates the idea of energy increase or decrease during a planetary flyby, and it leads to the correct conclusions.

$Q$ is at the location $\left(x_{1}(t), y_{1}(t)\right)$. The force is the usual inverse square attraction with potential function $U=\mu / r$ or with potential energy $V=-\mu / r$. The whole system is referred to an inertial system of reference with origin $O$ (Figure 1).

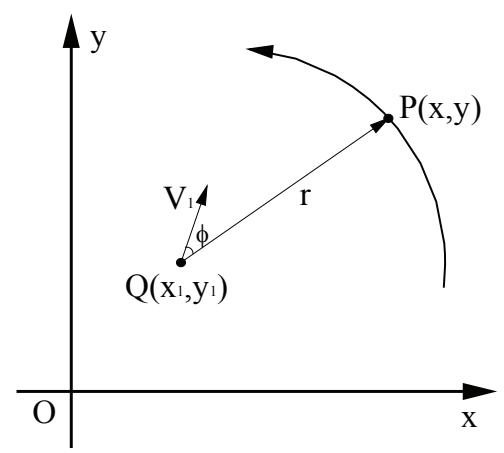

Figure 1 - A close approach between $P$ and $Q$.

The equations of motion of $P$ with respect to $Q$ are:

$$
\ddot{x}=\frac{\partial U}{\partial x}=-\mu \frac{x}{r^{3}} ; \quad \ddot{y}=\frac{\partial U}{\partial y}=-\mu \frac{y}{r^{3}},
$$

where

$$
r^{2}=\left(x-x_{1}\right)^{2}+\left(y-y_{1}\right)^{2} .
$$


The question is now the variation of the energy

$$
E=\frac{1}{2}\left(\dot{x}^{2}+\dot{y}^{2}\right)-U(r(x, y, t))
$$

of the particle at $P$. This is obtained with the use of the energy equation

$$
\frac{d E}{d t}=\frac{\partial E}{\partial t}=\frac{\partial V}{\partial t}=\frac{\mu}{r^{2}} \frac{\partial r}{\partial t},
$$

because the terms that are not explicitly dependent on the time are zero. However, we have

$$
r \frac{\partial r}{\partial t}=-\left(x-x_{1}\right) \frac{\partial x_{1}}{\partial t}-\left(y-y_{1}\right) \frac{\partial y_{1}}{\partial t}=-\left(x-x_{1}\right) \dot{x}_{1}-\left(y-y_{1}\right) \dot{y}_{1} .
$$

Therefore, the energy equation is:

$$
\frac{d E}{d t}=-\frac{\mu}{r^{3}}\left[\left(x-x_{1}\right) \dot{x}_{1}+\left(y-Y_{1}\right) \dot{y}_{1}\right] .
$$

In the brackets it is recognized the dot product of the relative position $\vec{r}=\overline{P Q}$ of $P$ with the absolute velocity vector $V_{1}\left(\dot{x}_{1}, \dot{y}_{1}\right)$ of the perturbing body $Q$. The energy equation can then be written as

$$
\frac{d E}{d t}=-\frac{\mu}{r^{3}} r V_{1} \cos (\phi)=-\frac{\mu V_{1}}{r^{2}} \cos (\phi) .
$$

From this equation it is possible to come to an important conclusion about the increase or decrease of the energy of the particle $P$. This essentially depends only on the factor $\cos (\phi)$ in the above equation: when $\phi$ is below $90^{\circ}$, the energy decreases and when $\phi$ is above $90^{\circ}$, the energy increases. This result can be summarized as follows: When $Q$ approaches: $E$ decreases, When $Q$ recedes: E increases.

\section{The Restricted Three-Body Problem}

The primary problem that is studied in this research is to find under what conditions a comet coming from outside the Solar System is captured. To solve this problem, it is assumed that the Solar System is formed only by three bodies: the Sun, a planet (Jupiter) and a third particle of negligible mass (the comet). It is also assumed that the total system (Sun + Jupiter + comet) obeys all the 
hypothesis of the planar restricted circular three-body problem, which means that: all the bodies are point masses; the Sun and Jupiter are in circular orbits around their mutual center of mass; the motion of the comet is governed by the two main bodies, but the comet does not interfere with their motion; the comet moves only in the orbital plane of the two primaries.

After these assumptions, the problem becomes to study the motion of the comet near the position where it crosses the line between the Sun and the planet Jupiter. It is enough to study its motion only near this point, because when the comet is far from Jupiter the system is governed by a two-body (Sun + comet) problem dynamics, that does not allow any change in energy. In particular, this research is looking for the energy of the comet before and after this crossing point, to detect under what conditions a comet is captured (change its energy from positive to negative). Since the initial conditions for the comet vary in a very large range, it is also possible to detect under what conditions a comet is expelled from the Solar System (change its energy from negative to positive) or have a modification in its energy without changing its type of orbit (a change in its energy that is not large enough to modify the sign of the energy). Figure 2 shows the geometry involved in the close approach and defines the basic variables used in this research.

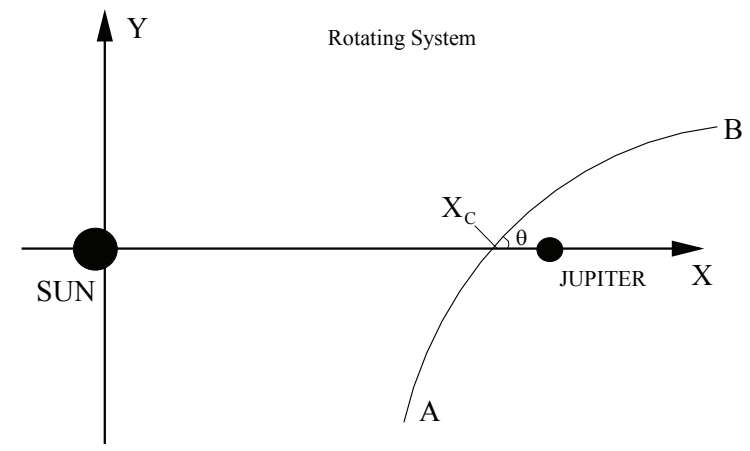

Figure 2 - Trajectory of the Comet when it is crossing the line Sun-Jupiter.

It shows that the comet leaves the point $A$, crosses the horizontal axis (the line between the Sun and the planet Jupiter) at the point $\left(X_{c}, 0\right)$, making an angle $\theta$ (measured from the horizontal axis in the counter-clock-wise direction) and goes to the point $B$. The points $A$ and $B$ are chosen in a such way that it is valid to neglect the influence of Jupiter at those points and, by consequence, it is known 
that the energy is constant after $B$ and before $A$. Under those assumptions, the procedure involved to solve this problem is:

i) To specify particular arbitrary values for the Jabobian constant, the ordinate of the crossing point $\left(X_{c}\right)$ and the angle $\theta$;

ii) Starting with the comet in the line Sun-Jupiter, integrate numerically its orbit forward in time until it reaches the point $B$;

iii) Starting again in the crossing line (point $\left(X_{c}, 0\right)$ ), integrate its orbit backward in time until the comet reaches the point $A$;

iv) At the points $A$ and $B$ the energy of the comet is calculated, and it is checked for the effects of this close approach with Jupiter.

The equations of motion used here are (using the canonical system of units) [33]:

$$
\ddot{x}-2 \dot{y}=x-\frac{\partial V}{\partial x}=\frac{\partial \Omega}{\partial x}, \quad \ddot{y}+2 \dot{x}=y-\frac{\partial V}{\partial y}=\frac{\partial \Omega}{\partial y}
$$

where $\Omega$ is the pseudo-potential function given by:

$$
\Omega=\frac{1}{2}\left(x^{2}+y^{2}\right)+\frac{(1-\mu)}{r_{1}}+\frac{\mu}{r_{2}}
$$

The Jacobi integral is given by:

$$
J=\frac{1}{2}\left(\dot{x}^{2}+\dot{y}^{2}\right)-\Omega(x, y)=\text { Const }
$$

It is also necessary to have equations to calculate the energy and the angular momentum of the comet. It can be done with the formulas:

$$
\begin{gathered}
E=\frac{(x+\dot{y})^{2}+(\dot{x}-y)^{2}}{2}-\frac{1-\mu}{r_{1}}+\frac{\mu}{r_{2}}, \\
C=x^{2}+y^{2}+x \dot{y}-y \dot{x}
\end{gathered}
$$

In those equations $r_{1}$ is the distance between the comet and the Sun and $r_{2}$ is the distance between the comet and Jupiter. With those equations, it is possible to build a numerical algorithm to solve the problem. It has the following steps: 
i) Give arbitrary values for the three parameters: $J, X_{c}, \theta$;

ii) With these values, compute the initial conditions in the rotating frame. The initial position is the point $\left(X_{c}, 0\right)$ and the initial velocity is $(V \cos \theta$, $V \sin \theta$ ), where $V=\sqrt{\dot{x}^{2}+\dot{y}^{2}}$ is calculated from equation (3);

iii) With these initial conditions, integrate the equations of motion forward in time until the distance between the planet Jupiter and the comet is greater than a specified limit $d_{J C}$ (the value 0.5 is used for this variable in the simulations made in this paper). At this point stop the numerical integration and calculate the energy $\left(E_{+}\right)$and the angular momentum $\left(C_{+}\right)$ after the encounter with Jupiter, from equations (4) and (5). The angular momentum gives the information about the sense of the orbit: direct or retrograde. Remember that it is assumed that the energy and the angular momentum is constant after this point, due to the fact that the perturbation from Jupiter is too small to disturb significantly the two-body character of the dynamics;

iv) Then go back to the initial conditions at the crossing point, and integrate the equations of motion backward in time, until the distance $d_{J C}$ is reached again. Then use the equations (4) and (5) to calculate the energy $\left(E_{-}\right)$and the angular momentum $\left(C_{-}\right)$before the encounter with Jupiter;

v) With those results, all the information required to calculate the change in energy $\left(E_{+}-E_{-}\right)$and angular momentum $\left(C_{+}-C_{-}\right)$due to the close approach with Jupiter are available.

With this algorithm available, it is possible to vary the given initial conditions (values for $J, X_{c}$ and $\theta$ ) in any desired range and study the effects of the close approach with Jupiter in the orbit of the comet.

\section{Results}

The results consist of plots that show what happens to the comet after the close encounter with the planet for a large range of given initial conditions. First of all it is necessary to classify all the close encounters between the planet and the 
comet, according to the change obtained in the orbit of the comet. The letters $H, B, E, h, b, c$ and $O$ are used for this classification. They are assigned to the orbits according to the rules showed in Table 1. It is necessary to emphasize that, due to the reversibility of the dynamical equations, all the changes obtained in the orbits are not permanent. So, the classification shown in Table 1 is valid for a limited amount of time and this limit depends on each case.

\begin{tabular}{|c|c|c|c|}
\hline Letter & Energy & Orbit before & Orbit after \\
\hline h & decrease & hyperbolic & hyperbolic \\
\hline b & decrease & elliptic & elliptic \\
\hline c & decrease & hyperbolic & elliptic \\
\hline H & increase & hyperbolic & hyperbolic \\
\hline B & increase & elliptic & elliptic \\
\hline E & increase & elliptic & hyperbolic \\
\hline
\end{tabular}

Table 1 - Rules of the assignment of letters to orbits.

The letter $O$ is used for an orbit that stays around Jupiter for a long time. In this case the comet became a temporary satellite of Jupiter. This is a case with little interest in the present research.

With those rules defined, the results consist of assigning one of those letters to a position in a two-dimensional diagram that has the parameter $X_{c}$ in the horizontal axis and the parameter $\theta$ in the vertical axis. One plot for each desired value of the Jacobian constant is made. To decide the best range of values for these three parameters, several exploratory simulations are made. It is noticed that for values of the Jacobian constant greater than 0.8 , the number of "captures" is very small, and for values below -1.45 a large number of orbits remain around Jupiter for a long time. Based on that, it is decided to use the range $-1.45<J<1.10$ for this parameter, and to make 18 plots in steps of 0.15 for $J$. Only some of them are shown here to save space, since they are all very similar.

For $X_{c}$, it is noticed that the values of interest (large number of captures) are between 0.8 and 1.2. Outside this range, the effect of the close approach is too small to be considered, due to the large distance from the comet to Jupiter. Taking that in consideration, the decision is in favor of a little wider range, and 
the ordinate of the crossing point is confined to the internal $0.7<X_{c}<1.3$.

For the angle $\theta$, it is noticed that there is a symmetry with respect to the line $\theta=90^{\circ}$. This symmetry comes from the fact that a trajectory with an angle $\theta$ is physically the same as a trajectory with an angle $\theta+180^{\circ}$ and the direction of time reversed. It means that the change in energy and angular momentum for these two trajectories has the same magnitude, and they differ only by the sign. Figure 3 shows this symmetry for the case $J=-1.3$.

It is possible to see clearly that the rules for the symmetry between the upper side of the diagram $\left(90^{\circ}<\theta<270^{\circ}\right)$ and the bottom side $\left(-90^{\circ}<\theta<90^{\circ}\right)$ is: $H \Leftrightarrow h, B \Leftrightarrow b$ and $E \Leftrightarrow c$. So, it is decided to keep $\theta$ in the interval $90^{\circ}<\theta<270^{\circ}$ (the upper side of the diagram) and the rest $\left(-90^{\circ}<\theta<90^{\circ}\right.$ ) can be derived by the symmetry rules.

From Figure 3 it is also easy to see the existence of several distinct regions. They are named with the numbers 1 to 5 , starting from the line $\theta=90^{\circ}$ and going in the sense of increasing $\theta$. The arithmetic symbols "-" and "+" are also used to indicate if the orbit crosses the Sun-Jupiter line in a point located between the two main bodies or outside Jupiter's orbit around the Sun. For example, orbit named " -1 " stands for an orbit that crosses the Sun-Jupiter line almost perpendicular to this line $\left(\theta\right.$ is a little more then $\left.90^{\circ}\right)$ in a point located between the Sun and Jupiter. For higher numbers the angle $\theta$ increases for each location of the crossing point. For orbits named with positive signs, the crossing point goes to the other side of Jupiter. Table 2 shows a sketch of each type of orbit.

A brief description of each region and border follows.

Region -1: It is formed by trajectories of type $H$. This is a type of orbit that is hyperbolic before and after the close encounter with Jupiter. There is a gain in energy in that maneuver, what makes the comet to leave the Solar System faster than it approaches it.

Region -2: It is formed by trajectories of type $E$. This is a type of orbit that is elliptic before and hyperbolic after the close encounter with Jupiter. There is a gain in energy in that maneuver, what makes a comet that belongs to the Solar System to escape from it. 
$\theta$

270 obbbbbbbbbbbbbbbBbbbBbbbbbbBbbbBbl bbbbbbbbbbbbbbbbbbbbbbbbbbbbbbbbbbl bbbbbbbbbbbbbbbbbbbbbbbbbbbbbbbbbbl bbbbbbbbbkbbbbbbbbbbbbbbbblbbbbbbbbt bbbbbbbbbbbbbbbbbbbbbbbbbbbbbbbbbbl bbbbbbbbbbbbbbbbbbbbbbbbbbbbbbbbbbl bbbbbbbbbbbbbbbbbbbb 5 'bbbbbbbbbbbl bbbbbbbbbbbbbbbbbbbk -5 , bbbbbbbbbbbl bbbbbbbbbbbbbbbbbbbbbbbbbbbbbbbbbbk bbbbbbbbbbbbbbbbbbbbbbbbbbbbbbbbbbl bbbbbbbbbbbbbbbbbbbbbbbbbbbbbbbbbb bbbbbbbbbbbbbbbbbbbbbbbbbbbbbbbbbl bbbbbbbbbbbbbbbbbbbbbbbbbbbbbbbbb bbbbbbbbbbbbbbbbbbbbbbbbbbbbbbb bbbbbbbbbbbbbbbbbbbbbbbbbbb

BbBbBBbBbBbbBbBbBBbbBbBbbBBbBBbBb BBBBBBBBBBBBBBBBBBBBBBBBBBBBBBBBBBBB BBBBBBBBBBBBBBBBBBBBBBBEBBBBBBBBBBE BBBBBBBBBBBBBBBBBBBBBBBBBBBBBBBEBBB BBBBBBBBBBBBBBBBBBBBBBBBBBBBBBBBBBBB BBBBBBBBBBBBB +5 BBBBBBBBBBBBBBBBBB

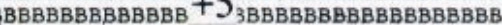
BBBBBBBBBBBBBBBBBBBBBBBBBBBBBBBBBBB 3BBBBBBBBBBBBBBBBBBBBBBBBBBBBBBBBBB BBBBBBBBBBBBBBBBBBBBBBBBBBBBBBBBBB BBBBBBBBBBBBBBBBBBBBBBBBBBBBBBBBB= (BBBBBBBBBBBBBBBBBBBBBBBR

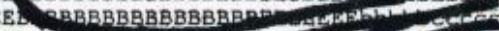

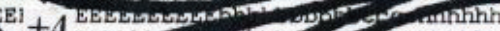

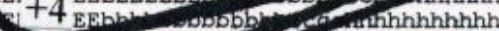

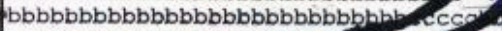
bbbbbbbbbbbbbbbbbbbbhhw

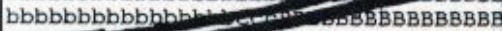
B

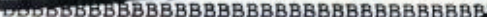
BBBBBBBBBBBBBBBBBBBBBBBBBBBBBBE BBBBBBBBBBBBBBBBBBBBBBBBBBBB ВВВВВВBВBВBВB - 3 ВВВВВВВВВВ BBBBBBBBBBBBBBBBBBBBB BBBBBBBBBBBBBBBBBBBBE BBBBBBBBBBBBBBBBBBB BBBBBBBBBBBBBBBBBB BBBBBBBBBBBBBBBBB BBBBBBBBBBBBBBBBE

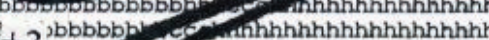
$+3$

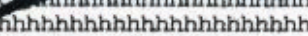

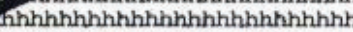

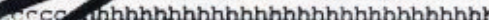

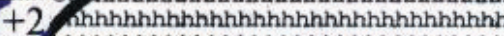

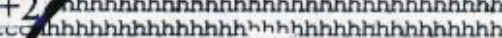

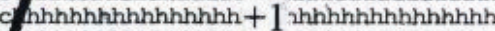

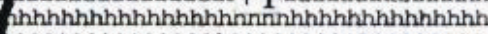

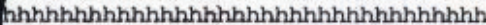

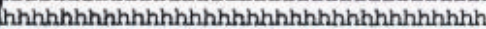

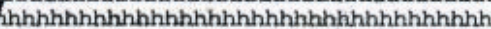

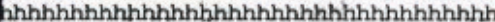

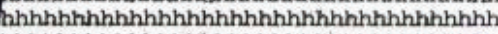

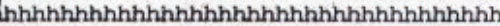

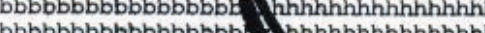
bbbbbbbbbbbbbbbbbb. \% hhhhhhhhhhhh bbbbbbbbbbbbbbbbbbbis hhhhhhhhhhht bbbbbbbbbbbbbbbbbbbbb o Whhhhhhhh bbbbbbbbbbbbbbbbbbbbbblon, hhihhhhl bbbbbbbbbbbbbbbbbbbbbbbbl

bbbbbbbbbbbbbbbbbbbbbbbbbbo bbbbbbbbbbbbbbbbbbbbbbbbbbbbb. ccec bbbbbbbbbbbbbbbbbbbbbbbbbbbbbbbb

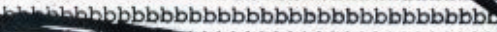

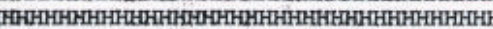

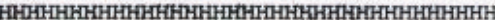

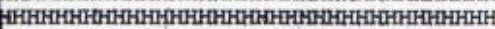

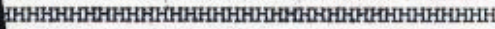

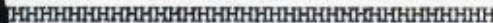

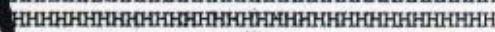

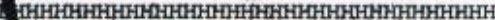

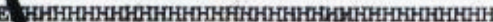

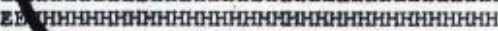

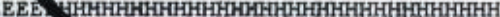
BEES \%

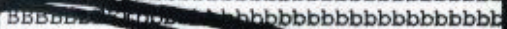

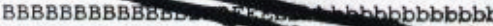

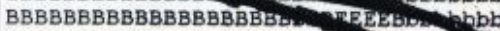

0 . BBBBBBBBBBBBBBBBBBBBBBBBBBBB 2\% EEEE BBBBBBBBBBBBBBBBBBBBBBBBBBBBBBB, BBBBBBBBBBBBBBBBBBBBABBBBBBBBBBBE BBBBBBBBBBBBBBBBBBBBBBBBBBBBBBBBB: BBBBBBBBBBBBBBBBBBBBBBBBBBBBBBBBBB BBBBBBBBBBBBBBBBBBBBBBBBBBBBBBBBBB BBBBBBBBBBBBBBBBBBBBBBBBBBBBBBBBBB BBBBBBBBBBBBBBBBBBBBBBBBBBBBBBBBBBBE BBBBBBBBBBBBBBBBBBBBBBBBBBBBBBBBBBE BBBBBBBBBBBBBBBBBBBBBBBBBBBBBBBBBBE BBBBBBBBBBBBBBBBBBBBBBBBBBBBBBBBBBE BBBBBBBBBBBBBBBBBBBBBBBBBBBBBBBBBBE BBBBBBBBBBBBBBBBBBBBBBBBBBBBBBBBBBB -90 , 1 BBBBBBBBBbBBBBBBBBBBBBBBBBBBBBBBBBE

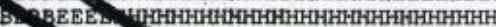

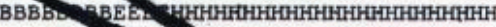

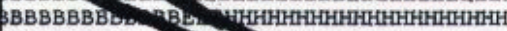

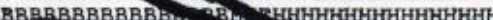
CCCCCCOS

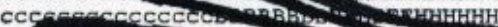
(1) Sbbbbbbbbbbbbbbbbbbbiow bbbbbbbbbbbbbbbbbbbbbbbbbbbbbbobot bbbbbbbbbbbbbbbbbbbbbbblbbbbbbbbbbb bbbbbbbbbbbbbbbbbbbbbbbbbbbbbbbbbbbbb bbbbbbbbbbbbbbbbbbbbbbbbbbbbbbbbbbb bbbbbbbbbbbbbbbbbbbbbbbbbbbbbbbbbbbb bbbbbbbbbbbbbbbbbbbbbbblbbbbbbbbbbbb bbbbbbbbbbbbbbbbbbbbbbbbbbbbbbbbbbb bbbbbbbbbbbbbbbbbbbbbbbbbbblabbbbbbb bbbbbbbbbbbbbbbbbbbbbbbbbbbbbbbbbbb bbbbbbbbbbbbbbbbbbbbbbbbbbbbbbbbbbb bbBbBBBBbBbbBbBBBbBBBbbbBBbbBBBbbbB
0.7

Figure 3 - The symmetry around the line $\theta=90^{\circ}(J=-1.3)$. 


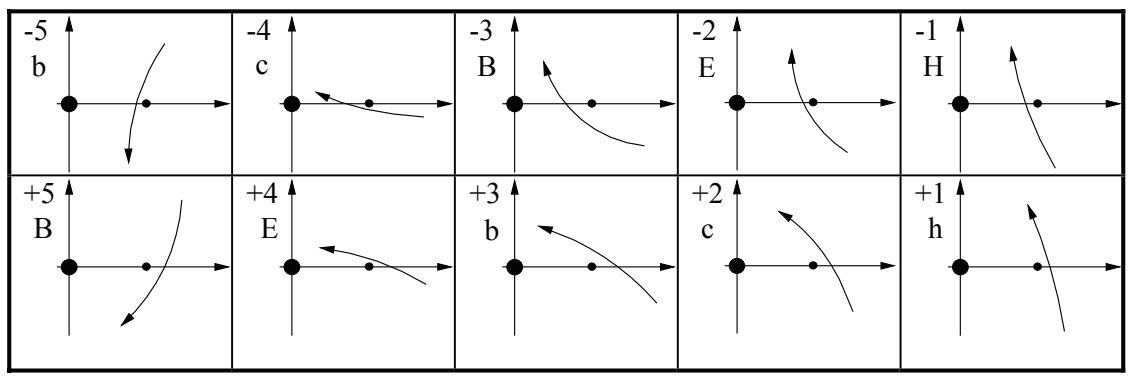

Table 2 - A sketch of each type of orbit found.

The border between the regions -1 and -2 : This border is formed by orbits that are parabolic before and hyperbolic after the close encounter with Jupiter. There is a gain in energy in that maneuver, what makes a comet that is in the limit of belonging or not to the Solar System to escape faster from it.

Region -3: $\quad$ It is formed by trajectories of type $B$. This is a type of orbit that is elliptic before and after the close encounter with Jupiter. There is a gain in energy in that maneuver, what makes the comet to increase its semi-major axis and to go to the direction of the outer Solar System.

The border between the regions -2 and -3 : This border is formed by orbits that are elliptic before and parabolic after the close encounter with Jupiter. There is a gain in energy in that maneuver, what makes a comet that belongs to the Solar System to go to the limit of belonging or not to it and to slowly escape to the interstellar space. This makes this case to be considered a maneuver of escape.

Region -4: It is formed by trajectories of type $c$. This is a type of orbit that is hyperbolic before and elliptic after the close encounter with Jupiter. There is a loss in energy in that maneuver, what makes a comet that does not belong to the Solar System to be captured and become part of it.

The border between the regions -3 and -4 : This border is formed by orbits that are parabolic before and elliptic after the close encounter with Jupiter. There is a loss in energy in that maneuver, what makes a comet that is in the limit of belonging or not to the Solar System to be completely captured and become part of it. This makes this case to be considered a maneuver of capture. This is also 
the line that divides gains and losses in energy. Below this line the comet always gains energy and above this line it always losses energy.

Region -5: It is formed by trajectories of type $b$. This is a type of orbit that is elliptic before and after the close encounter with Jupiter. There is a loss in energy in that maneuver, what makes a comet to decrease its semi-major axis and go to the direction of the interior of the Solar System.

The border between the regions -4 and -5 : This border is formed by orbits that are parabolic before and elliptic after the close encounter with Jupiter. There is a loss in energy in that maneuver, what makes a comet that is in the limit of belonging or not to the Solar System to be completely captured and become part of it. This makes this case to be considered a maneuver of capture.

Region +1: It is formed by trajectories of type $h$. This is a type of orbit that is hyperbolic before and after the close encounter with Jupiter. There is a loss in energy in that maneuver, what makes the comet to leave the Solar System slower than it approaches it.

Region +2: $\quad$ It is formed by trajectories of type $c$. This is a type of orbit that is hyperbolic before and elliptic after the close encounter with Jupiter. There is a loss in energy in that maneuver, what makes a comet that does not belong to the Solar System to be captured and become part of it.

Region +3: $\quad$ It is formed by trajectories of type $b$. This is a type of orbit that is elliptic before and after the close encounter with Jupiter. There is a loss in energy in that maneuver, what makes a comet to decrease its semi-major axis and go to the direction of the interior of the Solar System.

Region +4: It is formed by trajectories of type $E$. This is a type of orbit that is elliptic before and hyperbolic after the close encounter with Jupiter. There is a gain in energy in that maneuver, what makes a comet that belongs to the Solar System to escape from it. 
Region +5: $\quad$ It is formed by trajectories of type $B$. This is a type of orbit that is elliptic before and after the close encounter with Jupiter. There is a gain in energy in that maneuver, what makes the comet to increase its semi-major axis and to go to the direction of the outer Solar System.

The border between the regions +1 and +2 : This border is formed by orbits that are hyperbolic before and parabolic after the close encounter with Jupiter. There is a loss in energy in that maneuver, what makes a comet that does not belong to the Solar System to go to the limit of belonging or not to it.

The border between the regions +2 and +3 : This border is formed by orbits that are parabolic before and elliptic after the close encounter with Jupiter. There is a loss in energy in that maneuver, what makes a comet that is in the limit of belonging or not to the Solar System to be captured and become part of it. This makes this case to be considered a maneuver of capture.

The border between the regions +3 and +4 : This border is formed by orbits that are elliptic before and parabolic after the close encounter with Jupiter. There is a gain in energy in that maneuver, what makes a comet that belongs to the Solar System to go to the limit of belonging or not to it. This makes this case to be considered a maneuver of escape. This is also the line that divides gains and losses in energy. Below this line the comet always losses energy and above this line it always gains energy.

The border between the regions +4 and +5 : This border is formed by orbits that are elliptic before and parabolic after the close encounter with Jupiter. There is a gain in energy in that maneuver, what makes a comet that belongs to the Solar System to go to the limit of belonging or not to it. This makes this case to be considered a maneuver of escape.

The horizontal line $\theta=90^{\circ}$ is formed by orbits that have no change in energy. The close encounter with Jupiter has no effect in the trajectory of the comet. This is also the line of symmetry of the figure. The orbits below this line are the same ones above it with a reverse of time.

The vertical line $X_{c}=1.0$ is the line that reverses gains and losses in energy. For a given $\theta$, the left side has orbits with gain in energy, the right side has orbits with loss in energy and vice-versa. This line is close to the position of Jupiter, what means that crossing is equivalent to move from one side to another 


\section{of Jupiter with respect to the Sun.}

\section{Figure 4 shows a series of diagrams covering the desired range for all the three} variables. Plots for some values of $\mathbf{J}$ are omitted, since they are very similar to each other.

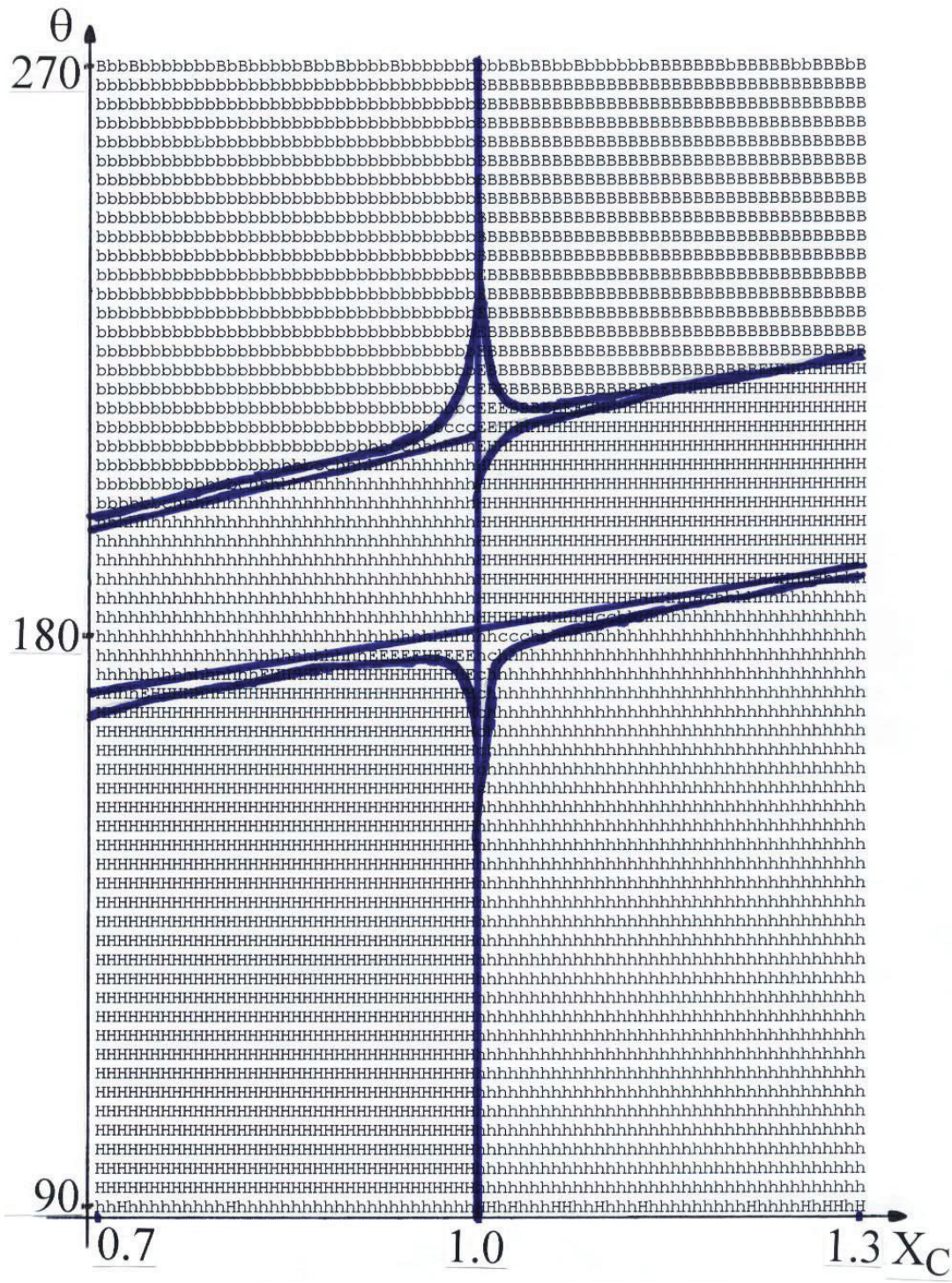

Figure $4.1-$ Results of the close approach for $J=-0.10$. 


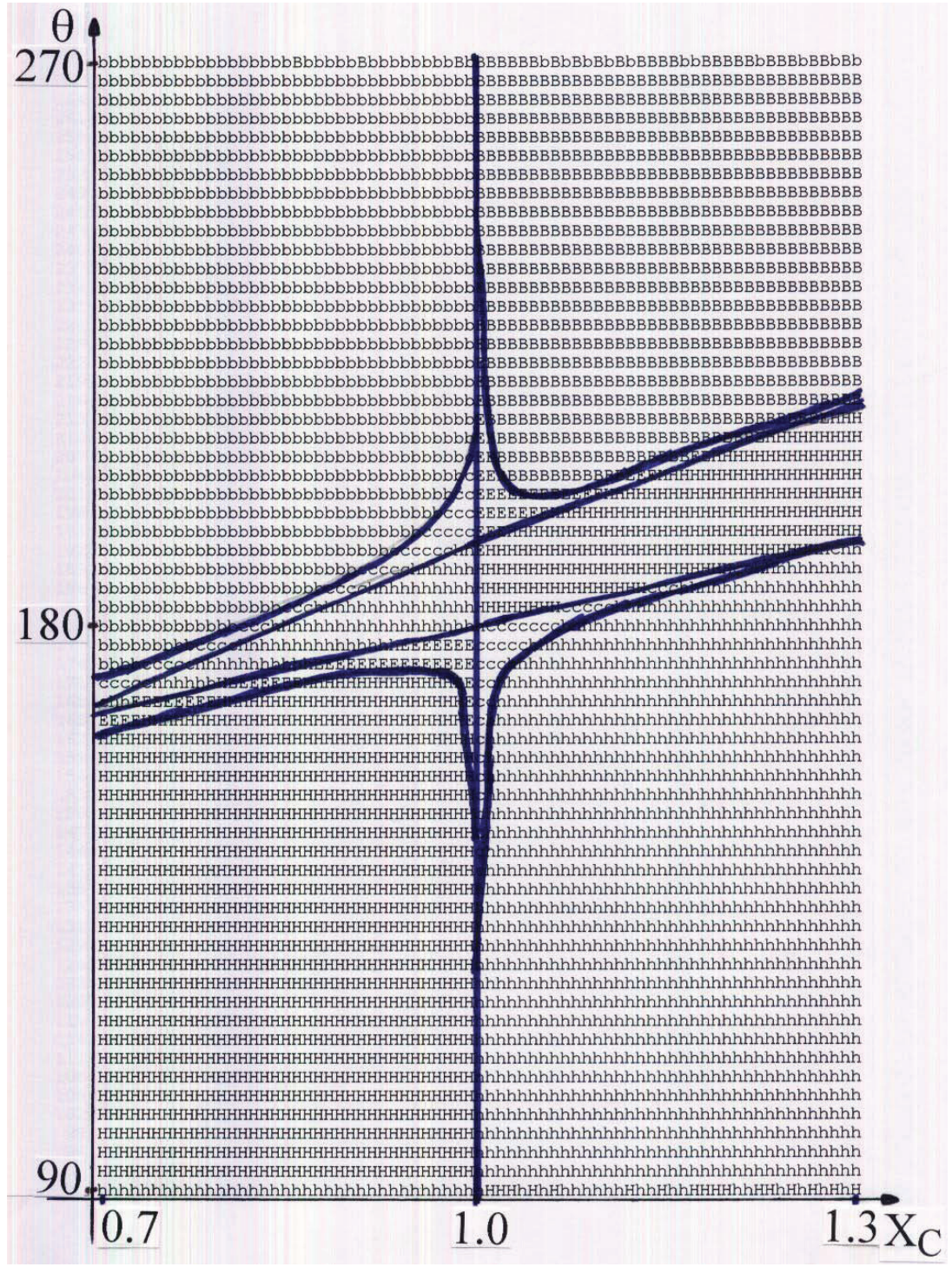

Figure 4.2 - Results of the close for $J=-0.70$.

To have a better understanding of the process, some of the trajectories are plotted in the rotating and fixed frame in Figure 5. Table 3 gives some of the numerical data for those trajectories, including the initial conditions.

Next, the occurrence of each event shown in Table 1 is counted. It is possible 


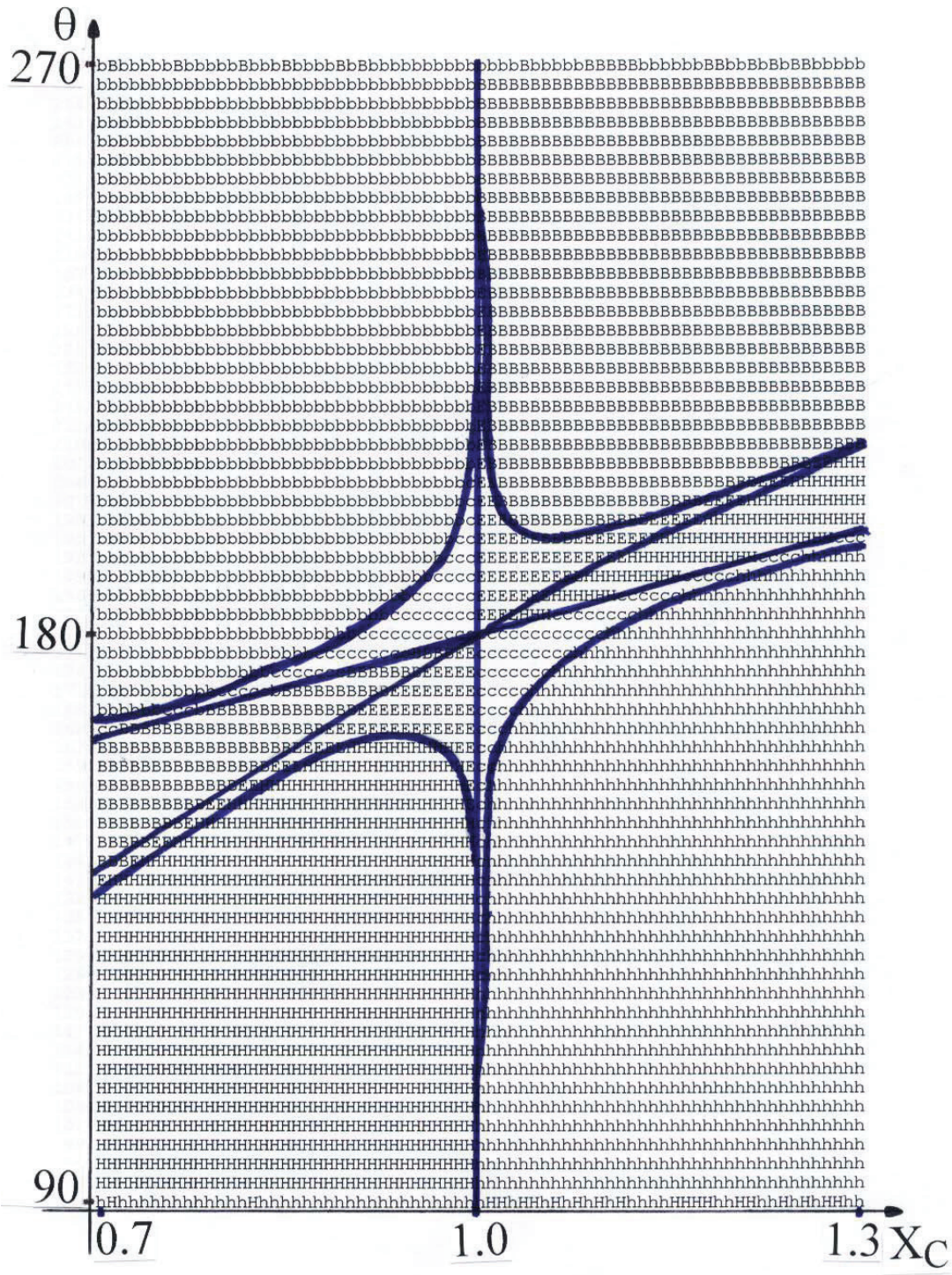

Figure 4.3 - Results of the close for $J=-1.00$.

to see that the number of captures and escapes (letters "c" and "E") has its maximum at $J=-1.15$ and it decreases fast when increasing or decreasing the value of the Jacobian constant. Assuming that comets traveling in this plane cross the horizontal axis with a homogeneous distribution of crossing angles $\theta$ and 


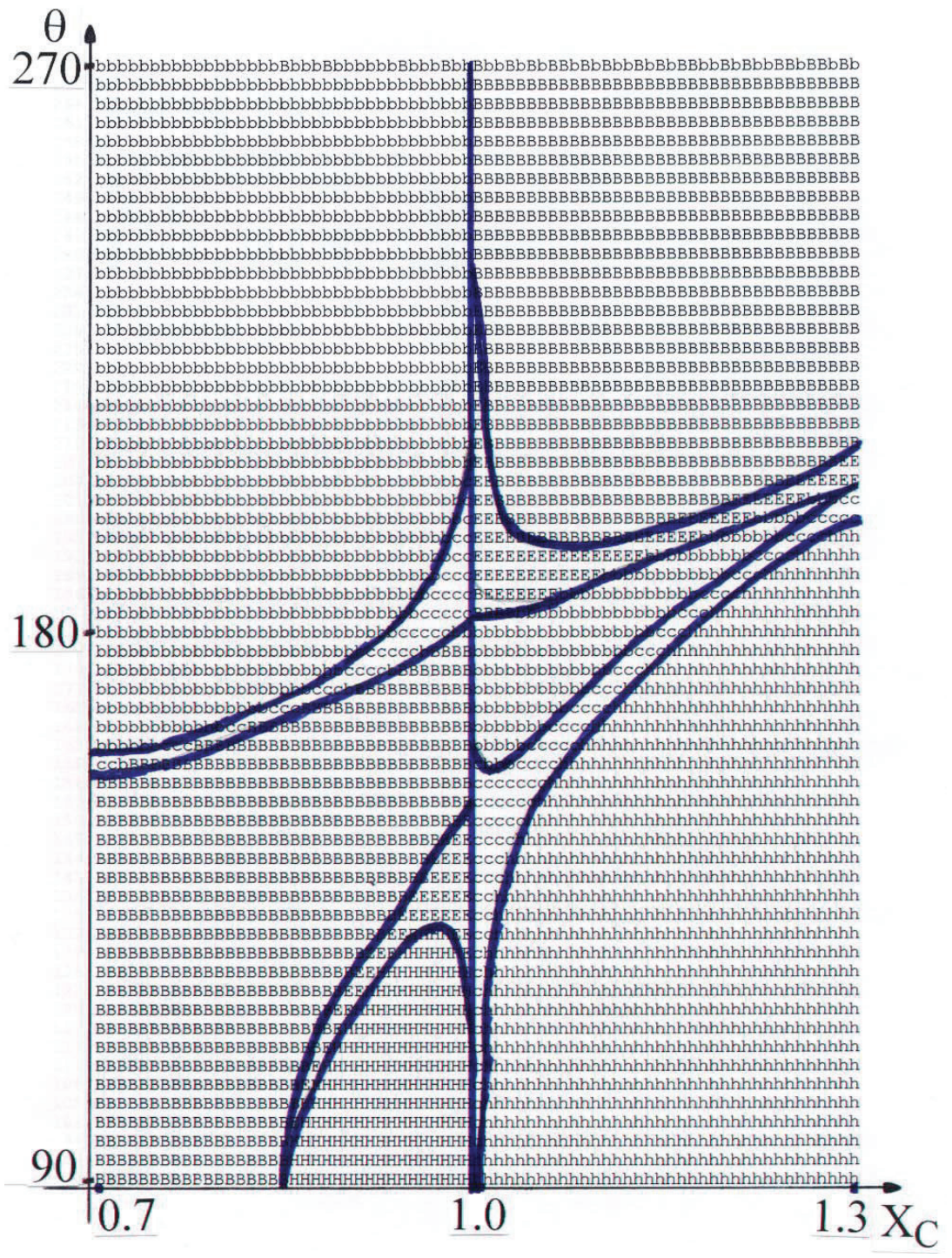

Figure 4.4 - Results of the close for $J=-1.30$.

ordinates of crossing points $X_{c}$, it is possible to estimate the probability of capture by counting the number of capture and escape orbits in this diagram (remember that an escape in the upper part of the diagram has a counterpart capture in the bottom part). Figure 6 shows the occurrence of each type of orbit as a function of 

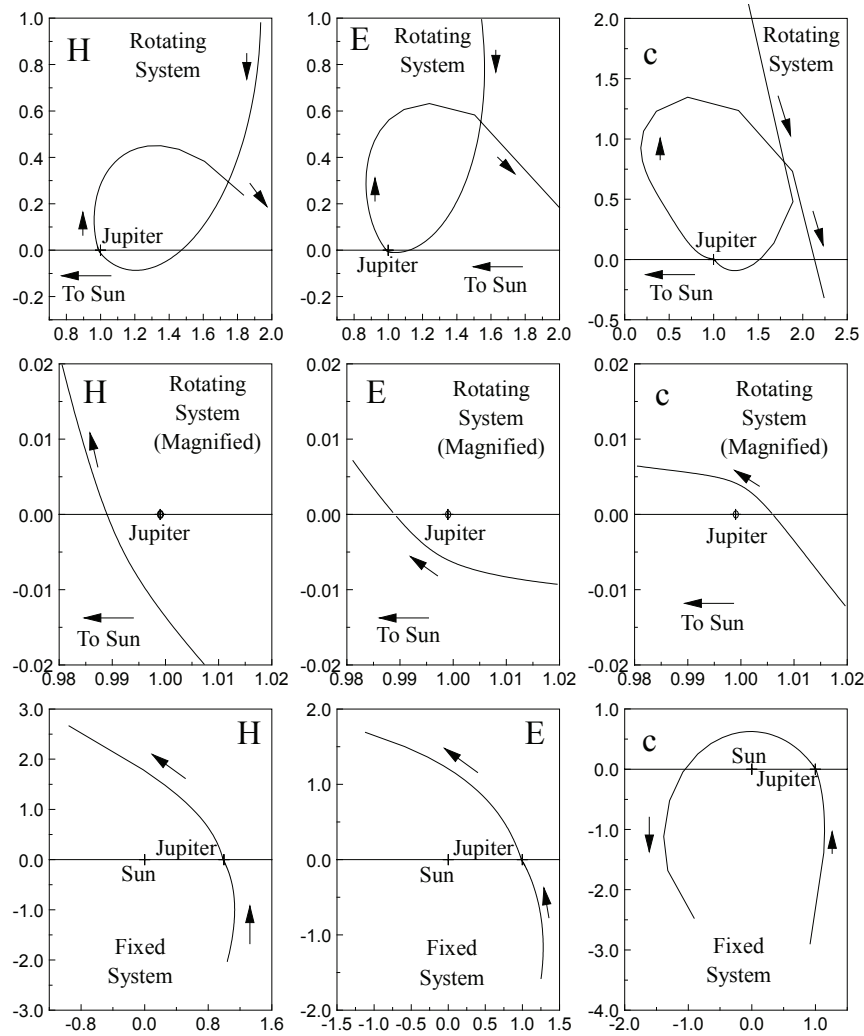

Figure 5 - Some examples of trajectories (Orbit $H$ is of type "-1", $E$ is "-2" and $c$ is "+2").

\begin{tabular}{|c|c|c|c|c|c|c|c|c|c|}
\hline Orbit & $\mathrm{J}$ & $X_{c}$ & $\theta$ & $E_{-}$ & $E_{+}$ & $\Delta E$ & $C_{-}$ & $C_{+}$ & $\Delta C$ \\
\hline $\mathrm{c}$ & -1.30 & 1.006 & 140.4 & 0.1690 & -0.2876 & -0.4566 & 1.4690 & 1.0124 & $\begin{array}{c}- \\
0.4566\end{array}$ \\
\hline $\mathrm{H}$ & -1.30 & 0.989 & 118.8 & 0.0987 & 0.2512 & 0.1525 & 1.3987 & 1.5512 & 0.1525 \\
\hline $\mathrm{E}$ & -1.30 & 0.989 & 140.4 & $\begin{array}{c}- \\
0.2232\end{array}$ & 0.1030 & 0.3262 & 1.0768 & 1.4030 & 0.3262 \\
\hline
\end{tabular}

Table 3 - Numerical data for the orbits plotted in Fig. 7.5.

the Jacobian constant and the probability of capture (total number of orbits type "c" and "E" divided by the total number of orbits). Remember that the above called "probability of capture" is the chance that a comet travelling in the orbital plane of the primaries and crossing the Sun-Jupiter line with $0.7<X_{c}<1.3$ is 
captured. It is not a chance that a generic comet coming to the Solar System is captured.
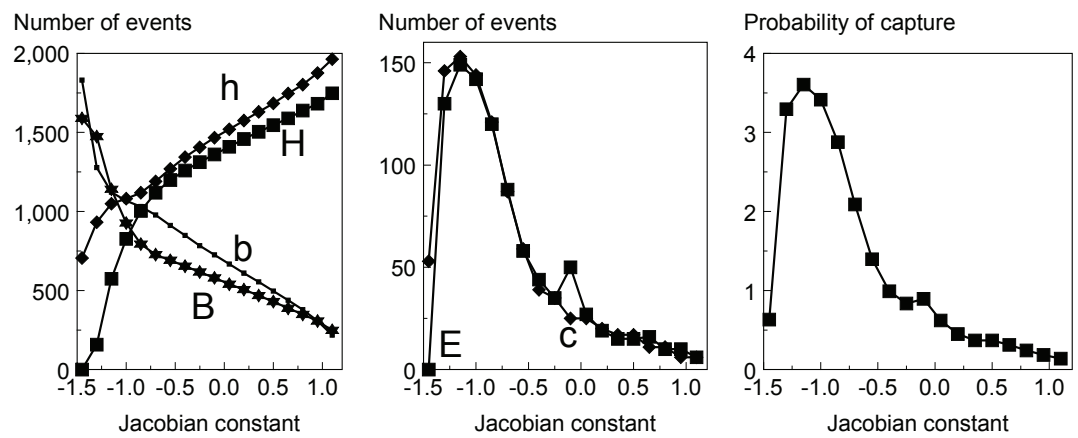

Figure 6 - Occurrence of each type of orbit and probability of capture as a function of $J$.

With this data available, it is possible to make several types of statistical analyses. In this research it is decided to make histograms that show the distribution of pericenter distance and semi-major axis (both in Astronomical Units - AU) of the captured comets. They are shown in Figure 7. It is possible to see that more than 95\% of the comets have a pericenter distance less than $5 \mathrm{AU}$, what makes them visible when passing by the pericenter of their orbits. It is also possible to see that about $70 \%$ of those comets have a semi-major axis less than $34.2 \mathrm{AU}$, what implies in an orbital period less than 200 years, and it makes them short-period comets. It is also included, in Figure 8, a detailed statistic of the percentage of short-period comets for each value of the Jacobian constant used. It is possible to see that there is a tendency in decreasing the percentage of short-period comets when the Jacobian constant is increased.

\section{Conclusions}

A numerical algorithm based in the circular planar restricted three-body problem is developed to study the motion of a comet in a Solar System formed by the Sun, a planet (Jupiter) and one comet. The effect of the close approach between the planet and the comet is studied for several values of the initial conditions for the comet. It is concluded that a comet can be captured by the Solar System due to this close approach, and the probability of capture goes from $0.2 \%$ to $3.6 \%$, 

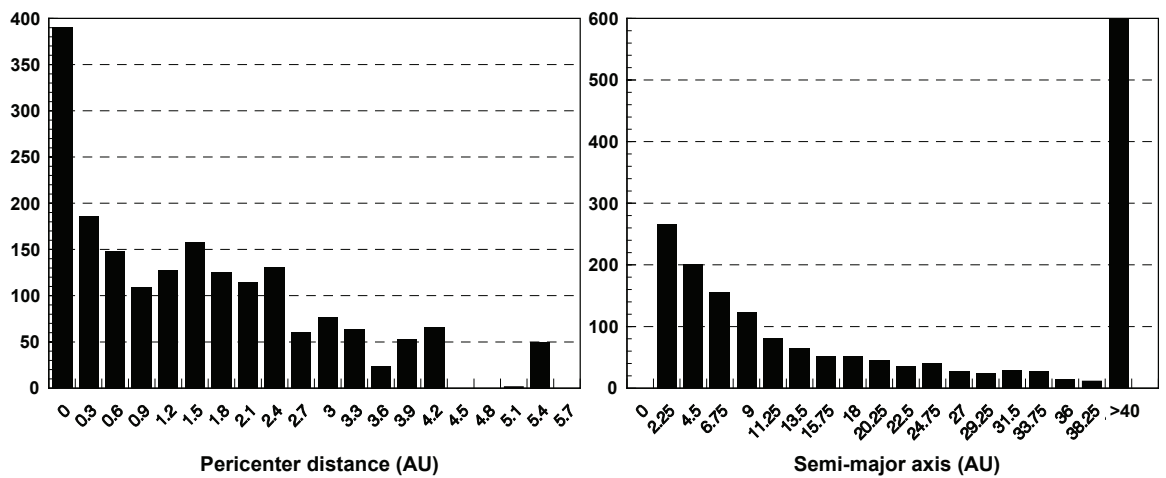

Figure 7 - Distribution of pericenter distance and semi-major axis for the captured comets.

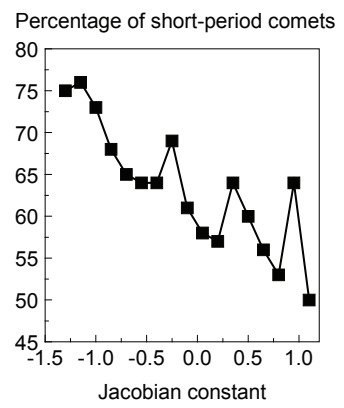

Figure 8 - Percentage of short-period comets after capture.

depending on the value of Jacobian constant of the comet. The details of this mechanism and some trajectories are shown. Some statistical analyses of the available data are also made. It is concluded that more than $95 \%$ of the captured comets have pericenter distance less than $5 \mathrm{AU}$ (what makes them visible) and that about $70 \%$ of them are short-period comets.

\section{REFERENCES}

[1] Laplace P., "Sur les Comètes," Connaissance des Temps. (1806).

[2] Yabushita S. and Hasegawa I., Monthly Notices Royal Astromical Society, 185 (1978), pp. 549.

[3] Oort J.H., "The Structure of the Cloud of Comets Surrounding the Solar System and a Hypothesis Concerning its Origin", Bulletin of the Astronomical Institutes of the Netherlands, 11 (1950), No. 91. 
[4] Bailey M.E., Ap. Space Sciences, 50 (1977), No. 3.

[5] Prado A.F.B.A., "Optimal Transfer and Swing-By Orbits in the Two- and Three-Body Problems", Ph.D. Dissertation, Univ. of Texas, Dept. of Aerospace Engineering and Engineering Mechanics, Austin, TX, USA, (1993).

[6] Russel H.N., "On the Origin of Periodic Comets", The Astronomical Journal, 32 (1920), No. 7, pp. 49-61.

[7] Woerkom A.J.J. Van, "On the Origin of Comets", Bulletin of the Astronomical Institutes of the Netherlands, 10 (1948), No. 399, pp. 445-472.

[8] Whipple F.L., The Astronomical Journal, 67 (1962), No. 1.

[9] Everhart E., Astrophysics Letters, 19 (1972), 131.

[10] Everhart E., Celestial Mechanics, 10 (1974), 35.

[11] Joss P.C., Astronomy and Astrophysics, 25 (1973), 271.

[12] Öpik E.J., Interplanetary Encounters, Elsevier, New York (1976).

[13] Marsden B.G., Sekanina Z. and Yeomans D.K., The Astronomical Journal, 78 (1973), 211.

[14] Yabushita S., Monthly Notices Royal Astromical Society, 187 (1979), 445.

[15] Hills J.G., The Astronomical Journal, 86 (1981), 1730.

[16] Fernández J.A., The Astronomical Journal, 87 (1982), 1318.

[17] Bailey M.E., Monthly Notices Royal Astromical Society, 211 (1984), 347.

[18] Bailey M.E., Advances in Space Research, 11 (1991), No. 6., pp. 43.

[19] Bailey M. E., Celestial Mechanics, 54 (1992), 49.

[20] Duncan M., Quinn T. and Tremaine S., The Astronomical Journal, 94 (1987), 1330.

[21] Bailey M.E. and Stagg C.R., Monthly Notices Royal Astromical Society, 235 (1988), 1.

[22] Bailey M.E. and Stagg C.R., Icarus, 86 (1990), 2.

[23] Petrosky T.Y. and Broucke R., Celestial Mechanics, 42 (1988), 53.

[24] Stagg C.R. and Bailey M.E., Monthly Notices Royal Astromical Society, 241 (1989), 507.

[25] Chirikov B.V. and Vecheslavov V.V., Astronomy and Astrophysics, 221 (1989), 146.

[26] Yabushita S. and Tsujii T., Monthly Notices Royal Astromical Society, 244 (1990), 663.

[27] Wetherill G.W., In: Newburn Jr. R.L., Neugebauer M. and Rahe J., eds, Proc. IAU Colloq. 116, Comets in the Post-Halley Era. Kluwer, Dordrecht, 1 (1991), p. 537.

[28] Emel'yanenko V.V., Celestial Mechanics, 54 (1992), 91.

[29] Levison H.F. and Duncan M.J., Icarus, 108 (1994), 18.

[30] Fernández J.A. and Gallardo T., Astronomy and Astrophysics, 281 (1994), 911. 
[31] Emel'yanenko V.V. and Bailey M.E., Earth, Moon and Planets, 72 (1996), 35.

[32] Emel'yanenko V.V. and Bailey M.E., Monthly Notices Royal Astromical Society, 298 (1998), 212.

[33] SzebehelyY, V.G., Theory of Orbits, Academic Press, New York (1967). 